\title{
The second edge-Wiener index of some composite graphs
}

\author{
Mahdieh Azari and Ali Iranmanesh
}




\title{
THE SECOND EDGE-WIENER INDEX OF SOME COMPOSITE GRAPHS
}

\author{
M. AZARI AND A. IRANMANESH
}

Received 11 October, 2013

\begin{abstract}
In this paper we study the behavior of the second edge-Wiener index under the join and corona product of graphs. Results are applied for some classes of graphs such as suspensions, bottlenecks, and thorny graphs.
\end{abstract}

2010 Mathematics Subject Classification: 05C12; 05C62; 92E10

Keywords: distance, second edge-Wiener index, join, corona

\section{INTRODUCTION}

In theoretical chemistry, the physico-chemical properties of chemical compounds are often modelled by means of molecular-graph-based structure-descriptors, which are also referred to as topological indices [14].

Let $G$ be a simple connected graph with vertex set $V(G)$ and edge set $E(G)$. The vertex version of the Wiener index is the first reported distance-based topological index which was introduced in 1947 by Wiener [16, 17], who used it for modeling the shape of organic molecules and for calculating several of their physico-chemical properties. The Wiener index $W(G)$ of $G$ is defined as:

$$
W(G)=\sum_{\{u, v\} \subseteq V(G)} d_{G}(u, v),
$$

where $d_{G}(u, v)$ is the shortest path distance between vertices $u$ and $v$ in $G$. Details on the Wiener index can be found in $[7,8,12,14,15]$.

Edge versions of the Wiener index based on the distance between all pairs of edges in a simple connected graph $G$ were introduced in 2009 [9]. Two possible distances between the edges $g=u v$ and $f=z t$ of a graph $G$ can be considered. Each of them gives rise to a corresponding edge-Wiener index. The first distance is the one based on the distance between the corresponding vertices in the line graph of $G$ and obviously, its related edge-Wiener index is equal to the ordinary Wiener index of the line graph of $G$. The second distance $d_{e \mid G}(g, f)$ between the edges $g=u v$ and 
$f=z t$ of the graph $G$ is defined as [9]:

$$
d_{e \mid G}(g, f)= \begin{cases}0 & g=f \\ \max \left\{d_{G}(u, z), d_{G}(u, t), d_{G}(v, z), d_{G}(v, t)\right\} & g \neq f\end{cases}
$$

Related to this distance, the second edge-Wiener index $W_{e}(G)$ of $G$ is defined as [9]:

$$
W_{e}(G)=\sum_{\{g, f\} \subseteq E(G)} d_{e \mid G}(g, f) .
$$

We refer the reader to $[6,10]$ for more information on the edge-Wiener indices.

The first edge-Wiener index of some composite graphs was computed before [1, 2,4]. In this paper, we are interested in the type of relationship that exists between the second edge-Wiener index of the join and corona product of graphs and their components. Results are applied for several classes of graphs by specializing components in these product graphs. So throughout the paper, by the edge-Wiener index of a graph $G$, we mean the second version, $W_{e}(G)$ and by distance between two edges $g$ and $f$ in $G$, we mean the second distance $d_{e \mid G}(g, f)$.

\section{PRELIMINARIES}

Let $N_{G}(u)$ denote the neighborhood of a vertex $u$ in $G$, i.e., the set of all vertices of $G$ adjacent with $u$. The degree of $u$ in $G$ is the cardinality of $N_{G}(u)$ and is denoted by $\operatorname{deg}_{G}(u)$. Let $\Delta(G)$ and $\Theta(G)$ denote the number of triangles in $G$ and the number of subgraphs of $G$ isomorphic to the 4-vertex complete graph $K_{4}$, respectively. It is easy to see that

$$
\begin{gathered}
\Delta(G)=\frac{1}{3} \sum_{u v \in E(G)}\left|N_{G}(u) \cap N_{G}(v)\right|, \\
\Theta(G)=\frac{1}{12} \sum_{u v \in E(G)} \sum_{z \in N_{G}(u) \cap N_{G}(v)}\left|N_{G}(u) \cap N_{G}(v) \cap N_{G}(z)\right| .
\end{gathered}
$$

Corresponding to each triangle in $G$, there are 3 pairs of adjacent edges which are at distance $1 \mathrm{in} G$. So the number of such pairs of edges in $G$ is equal to $3 \Delta(G)$. Also, corresponding to each subgraph of $G$ isomorphic to $K_{4}$, there are 3 pairs of nonadjacent edges which are at distance 1 in $G$. So the number of such pairs of edges in $G$ is equal to $3 \Theta(G)$. Hence, the total number of pairs of edges which are at distance 1 in $G$ is equal to $3(\Delta(G)+\Theta(G))$.

Let $x$ be a vertex of $G$ and $g=u v$ be an edge of $G$. We define the distance $D_{G}(x, g)$ between the vertex $x$ and the edge $g$ of the graph $G$ as [5]:

$$
D_{G}(x, g)=\max \left\{d_{G}(x, u), d_{G}(x, v)\right\} .
$$


The vertex-edge Wiener index $W_{v e}(G)$ of $G$ is defined as the sum of such distances over all vertices $x \in V(G)$ and edges $g \in E(G)$ [5]:

$$
W_{v e}(G)=\sum_{x \in V(G)} \sum_{g \in E(G)} D(x, g \mid G) .
$$

This index was studied in more details in [1,3] under the name $\operatorname{Max}(G)$. We refer the reader to these references for more information on the vertex-edge Wiener indices and for explicit formulas for $W_{v e}(G)$ of several classes of graphs.

\section{MAIN RESULTS}

Throughout this section, let $G_{1}$ and $G_{2}$ be two simple connected graphs and $n_{i}$ and $e_{i}$ denote the numbers of vertices and edges of $G_{i}$, respectively, where $i \in\{1,2\}$. Our aim is to compute the edge-Wiener index of join and corona product of $G_{1}$ and $G_{2}$.

\subsection{Join}

The join $G_{1}+G_{2}$ of graphs $G_{1}$ and $G_{2}$ is defined as the graph with the vertex set $V\left(G_{1}\right) \cup V\left(G_{2}\right)$ and the edge set

$$
E\left(G_{1}+G_{2}\right)=E\left(G_{1}\right) \cup E\left(G_{2}\right) \cup S,
$$

where $S=\left\{u_{1} u_{2} \mid u_{1} \in V\left(G_{1}\right), u_{2} \in V\left(G_{2}\right)\right\}$. All distinct vertices of $G_{1}+G_{2}$ are either at distance 1 or 2 . The vertices at distance 2 are precisely those of $G_{1}$ that are not adjacent in $G_{1}$, and those of $G_{2}$ that are not adjacent in $G_{2}$. So all distinct edges of $G_{1}+G_{2}$ are either at distance 1 or 2 . The join of two graphs is also known as their sum. Its definition can be extended inductively to more than two graphs in a straightforward manner. It is a commutative operation and hence both its components will appear symmetrically in any formula including distance-based invariants.

Theorem 1. Let $G_{1}$ and $G_{2}$ be two simple connected graphs. Then

$$
\begin{aligned}
W_{e}\left(G_{1}+G_{2}\right)= & 2\left(\begin{array}{c}
n_{1} n_{2} \\
2
\end{array}\right)+2\left(\begin{array}{c}
e_{1} \\
2
\end{array}\right)+2\left(\begin{array}{c}
e_{2} \\
2
\end{array}\right)+e_{1} n_{2}\left(2 n_{1}-3\right)+n_{1} e_{2}\left(2 n_{2}-3\right) \\
& -e_{1} e_{2}-3\left(n_{2}+1\right) \Delta\left(G_{1}\right)-3\left(n_{1}+1\right) \Delta\left(G_{2}\right)-3\left(\Theta\left(G_{1}\right)+\Theta\left(G_{2}\right)\right) .
\end{aligned}
$$

Proof. Let $Q$ be the set of all pairs of edges of $G_{1}+G_{2}$. We partition $Q$ into six disjoint sets as follows:

$$
\begin{aligned}
& Q_{1}=\left\{\{g, f\} \mid g, f \in E\left(G_{1}\right)\right\} ; \\
& Q_{2}=\left\{\{g, f\} \mid g, f \in E\left(G_{2}\right)\right\} ; \\
& Q_{3}=\left\{\{g, f\} \mid g \in E\left(G_{1}\right), f \in E\left(G_{2}\right)\right\} ; \\
& Q_{4}=\left\{\{g, f\} \mid g \in E\left(G_{1}\right), f \in S\right\} ; \\
& Q_{5}=\left\{\{g, f\} \mid g \in E\left(G_{2}\right), f \in S\right\} ; \\
& Q_{6}=\{\{g, f\} \mid g, f \in S\} .
\end{aligned}
$$

The edge-Wiener index of $G_{1}+G_{2}$ is obtained by summing the contributions of all 
pairs of edges over those six sets. We proceed to evaluate their contributions in order of increasing complexity.

The case of $Q_{3}$ is the simplest. Let $\{g, f\} \in Q_{3}$, where $g=u_{1} v_{1} \in E\left(G_{1}\right)$ and $f=u_{2} v_{2} \in E\left(G_{2}\right)$. Then

$$
\begin{aligned}
d_{e \mid G_{1}+G_{2}}(g, f)= & \max \left\{d_{G_{1}+G_{2}}\left(u_{1}, u_{2}\right), d_{G_{1}+G_{2}}\left(u_{1}, v_{2}\right), d_{G_{1}+G_{2}}\left(v_{1}, u_{2}\right),\right. \\
& \left.d_{G_{1}+G_{2}}\left(v_{1}, v_{2}\right)\right\} \\
= & \max \{1,1,1,1\}=1 .
\end{aligned}
$$

There are $e_{1} e_{2}$ such pairs of edges in $Q_{3}$ and each of them contributes 1 to the edgeWiener index. Hence, the total contribution of pairs from $Q_{3}$ is equal to $e_{1} e_{2}$.

The set $Q_{6}$ contains pairs of edges from $S$. Let $\{g, f\} \in Q_{6}$ and $g=u_{1} u_{2}$, $f=v_{1} v_{2}$, where $u_{1}, v_{1} \in V\left(G_{1}\right), u_{2}, v_{2} \in V\left(G_{2}\right)$. Then

$$
\begin{aligned}
d_{e \mid G_{1}+G_{2}}(g, f)= & \max \left\{d_{G_{1}+G_{2}}\left(u_{1}, v_{1}\right), d_{G_{1}+G_{2}}\left(u_{1}, v_{2}\right), d_{G_{1}+G_{2}}\left(u_{2}, v_{1}\right),\right. \\
& \left.d_{G_{1}+G_{2}}\left(u_{2}, v_{2}\right)\right\} \\
= & \max \left\{d_{G_{1}+G_{2}}\left(u_{1}, v_{1}\right), 1,1, d_{G_{1}+G_{2}}\left(u_{2}, v_{2}\right)\right\} .
\end{aligned}
$$

It is easy to see that, if $u_{1}=v_{1}, u_{2} v_{2} \in E\left(G_{2}\right)$ or $u_{2}=v_{2}, u_{1} v_{1} \in E\left(G_{1}\right)$ or $u_{1} v_{1} \in$ $E\left(G_{1}\right), u_{2} v_{2} \in E\left(G_{2}\right)$ then $d_{e \mid G_{1}+G_{2}}(g, f)=1$, otherwise $d_{e \mid G_{1}+G_{2}}(g, f)=2$.

The total number of pairs of edges in $Q_{6}$ is equal to $\left(\begin{array}{c}n_{1} n_{2} \\ 2\end{array}\right)$. Among them there are $n_{1} e_{2}+n_{2} e_{1}+2 e_{1} e_{2}$ pairs that contribute 1 to the edge-Wiener index, and all other pairs contribute 2. Hence the total contribution of pairs from $Q_{6}$ is equal to

$$
2\left(\begin{array}{c}
n_{1} n_{2} \\
2
\end{array}\right)-n_{1} e_{2}-n_{2} e_{1}-2 e_{1} e_{2} .
$$

Now, we compute the contribution of pairs from $Q_{4}$. Let $\{g, f\} \in Q_{4}$ and $g=$ $u_{1} v_{1} \in E\left(G_{1}\right)$ and $f=z_{1} u_{2} \in S$, where $u_{1}, v_{1}, z_{1} \in V\left(G_{1}\right), u_{2} \in V\left(G_{2}\right)$. Then

$$
\begin{aligned}
d_{e \mid G_{1}+G_{2}}(g, f)= & \max \left\{d_{G_{1}+G_{2}}\left(u_{1}, z_{1}\right), d_{G_{1}+G_{2}}\left(u_{1}, u_{2}\right), d_{G_{1}+G_{2}}\left(v_{1}, z_{1}\right),\right. \\
& \left.d_{G_{1}+G_{2}}\left(v_{1}, u_{2}\right)\right\} \\
= & \max \left\{d_{G_{1}+G_{2}}\left(u_{1}, z_{1}\right), 1, d_{G_{1}+G_{2}}\left(v_{1}, z_{1}\right), 1\right\} .
\end{aligned}
$$

It is easy to see that, if $z_{1}=u_{1}$ or $z_{1}=v_{1}$ or $u_{1} z_{1}, v_{1} z_{1} \in E\left(G_{1}\right)$ then $d_{e \mid G_{1}+G_{2}}(g, f)=1$, otherwise $d_{e \mid G_{1}+G_{2}}(g, f)=2$.

The total number of pairs from $Q_{4}$ is equal to $e_{1} n_{1} n_{2}$. Among them there are $2 e_{1} n_{2}+3 n_{2} \Delta\left(G_{1}\right)$ pairs that contribute 1 to the edge-Wiener index, and all other pairs contribute 2 . Hence the total contribution of pairs from $Q_{4}$ is equal to

$$
2 e_{1} n_{1} n_{2}-2 e_{1} n_{2}-3 n_{2} \Delta\left(G_{1}\right) .
$$

By symmetry, the total contribution of pairs from $Q_{5}$ is equal to

$$
2 n_{1} n_{2} e_{2}-2 n_{1} e_{2}-3 n_{1} \Delta\left(G_{2}\right) .
$$


It remains to compute the contributions of $Q_{1}$ and $Q_{2}$. Let $\{g, f\} \in Q_{1}$, where $g=u_{1} v_{1}, f=z_{1} t_{1}$. Then

$$
\begin{gathered}
d_{e \mid G_{1}+G_{2}}(g, f)=\max \left\{d_{G_{1}+G_{2}}\left(u_{1}, z_{1}\right), d_{G_{1}+G_{2}}\left(u_{1}, t_{1}\right), d_{G_{1}+G_{2}}\left(v_{1}, z_{1}\right),\right. \\
\left.d_{G_{1}+G_{2}}\left(v_{1}, t_{1}\right)\right\} .
\end{gathered}
$$

By definition of $G_{1}+G_{2}$, the distances $d_{G_{1}+G_{2}}\left(u_{1}, z_{1}\right), d_{G_{1}+G_{2}}\left(u_{1}, t_{1}\right)$, $d_{G_{1}+G_{2}}\left(v_{1}, z_{1}\right)$ and $d_{G_{1}+G_{2}}\left(v_{1}, t_{1}\right)$ are equal to 0,1 or 2 , so $d_{e \mid G_{1}+G_{2}}(g, f)$ is either equal to 1 or 2 . The total number of pairs in $Q_{1}$ is equal to $\left(\begin{array}{c}e_{1} \\ 2\end{array}\right)$. As mentioned before, $3\left(\Delta\left(G_{1}\right)+\Theta\left(G_{1}\right)\right)$ pairs contribute 1 to the edge-Wiener index, and all other pairs contribute 2 . Hence the total contribution of pairs from $Q_{1}$ is equal to

$$
2\left(\begin{array}{c}
e_{1} \\
2
\end{array}\right)-3\left(\Delta\left(G_{1}\right)+\Theta\left(G_{1}\right)\right) .
$$

Again, the total contribution of $Q_{2}$ is obtained by the symmetry as

$$
2\left(\begin{array}{c}
e_{2} \\
2
\end{array}\right)-3\left(\Delta\left(G_{2}\right)+\Theta\left(G_{2}\right)\right) .
$$

Now, the formula of the Theorem follows by adding the contributions of $Q_{1}, \ldots$, $Q_{6}$ and simplifying the resulting expression.

As expected, $G_{1}$ and $G_{2}$ appear symmetrically in the above formula. It is interesting to note that the formula does not depend on the connectivity of $G_{1}$ and $G_{2}$. That allows us to compute the edge-Wiener index of joins of graphs that are not themselves connected.

\subsection{Corona product}

The corona product $G_{1} \circ G_{2}$ of graphs $G_{1}$ and $G_{2}$ is obtained by taking one copy of $G_{1}$ and $n_{1}$ copies of $G_{2}$, and joining all vertices of the $i$-th copy of $G_{2}$ to the $i$-th vertex of $G_{1}$ for $i=1,2, \cdots, n_{1}$. Unlike join, corona is a non-commutative operation, and its component graphs appear in markedly asymmetric roles. More formally, we denote the copy of $G_{2}$ related to the vertex $x \in V\left(G_{1}\right)$ by $G_{2, x}$ and the edge set of $G_{2, x}$ by $S_{2, x}$. By definition of $G_{1} \circ G_{2}$, the distance between two distinct vertices $u, v \in V\left(G_{1} \circ G_{2}\right)$ is given by:

$$
d_{G_{1} \circ G_{2}}(u, v)= \begin{cases}d_{G_{1}}(u, v) & u, v \in V\left(G_{1}\right) \\ d_{G_{1}}(u, x)+1 & u \in V\left(G_{1}\right), v \in V\left(G_{2, x}\right) \\ 1 & u v \in S_{2, x} \\ 2 & u, v \in V\left(G_{2, x}\right), u v \notin S_{2, x} \\ d_{G_{1}}(x, y)+2 & u \in V\left(G_{2, x}\right), v \in V\left(G_{2, y}\right), x \neq y\end{cases}
$$

Theorem 2. Let $G_{1}$ and $G_{2}$ be two simple connected graphs. Then

$W_{e}\left(G_{1} \circ G_{2}\right)=W_{e}\left(G_{1}\right)+\left(n_{2}+e_{2}\right)^{2} W\left(G_{1}\right)+\left(n_{2}+e_{2}\right) W_{v e}\left(G_{1}\right)-6 n_{1} \Delta\left(G_{2}\right)$ 


$$
\begin{aligned}
& -3 n_{1} \Theta\left(G_{2}\right)+2\left(\begin{array}{c}
n_{1} n_{2} \\
2
\end{array}\right)+2\left(\begin{array}{c}
n_{1} e_{2} \\
2
\end{array}\right)+n_{1} e_{1}\left(n_{2}+e_{2}\right) \\
& +n_{1} e_{2}\left(2 n_{1} n_{2}-3\right) .
\end{aligned}
$$

Proof. It is obvious that the graph $G_{1} \circ G_{2}$ has $e_{1}+n_{1} e_{2}+n_{1} n_{2}$ edges. We partition the edge set of $G_{1} \circ G_{2}$ into three sets. The first one is the edge set of $G_{1}, S_{1}=$ $E\left(G_{1}\right)$, the second one contains all edges in all copies of $G_{2}, S_{2}=\bigcup_{x \in V\left(G_{1}\right)} S_{2, x}$, and the third one contains all edges with one end in $G_{1}$ and the other end in some copy of $G_{2}, S_{3}=\bigcup_{x \in V\left(G_{1}\right)} S_{3, x}$, where $S_{3, x}=\left\{e \mid e=u x, u \in V\left(G_{2, x}\right)\right\}$.

Now we start to compute the distances between the edges of these three sets. We consider the following six cases:

Case 1. $\{g, f\} \subseteq S_{1}$.

It is obvious that $d_{e \mid G_{1} \circ G_{2}}(g, f)=d_{e \mid G_{1}}(g, f)$, so

$$
W_{1}=\sum_{\{g, f\} \subseteq S_{1}} d_{e \mid G_{1} \circ G_{2}}(g, f)=W_{e}\left(G_{1}\right) .
$$

Case 2. $\{g, f\} \subseteq S_{2}, g \in S_{2, x}$ and $f \in S_{2, y}$.

First, consider the case $x=y$ and let $g=u_{2, x} v_{2, x}, f=z_{2, x} t_{2, x} \in S_{2, x}$. Then

$$
\begin{gathered}
d_{e \mid G_{1} \circ G_{2}}(g, f)=\max \left\{d_{G_{1} \circ G_{2}}\left(u_{2, x}, z_{2, x}\right), d_{G_{1} \circ G_{2}}\left(u_{2, x}, t_{2, x}\right), d_{G_{1} \circ G_{2}}\left(v_{2, x}, z_{2, x}\right),\right. \\
\left.d_{G_{1} \circ G_{2}}\left(v_{2, x}, t_{2, x}\right)\right\} .
\end{gathered}
$$

Clearly, the above four distances are equal to 0,1 or 2 . So $d_{e \mid G_{1} \circ G_{2}}(g, f)=1$ or 2 . In this case, the vertex $x$ and its related copy, $G_{2, x}$, form a copy of $K_{1}+G_{2}$. So by the same reasoning as in the proof of Theorem 1, we obtain:

$$
\sum_{\{g, f\} \subseteq S_{2, x}} d_{e \mid G_{1} \circ G_{2}}(g, f)=2\left(\begin{array}{c}
e_{2} \\
2
\end{array}\right)-3\left(\Delta\left(G_{2}\right)+\Theta\left(G_{2}\right)\right) .
$$

Now let $x \neq y$ and $g=u_{2, x} v_{2, x}$ and $f=u_{2, y} v_{2, y}$. Then

$$
\begin{aligned}
d_{e \mid G_{1} \circ G_{2}}(g, f)= & \max \left\{d_{G_{1} \circ G_{2}}\left(u_{2, x}, u_{2, y}\right), d_{G_{1} \circ G_{2}}\left(u_{2, x}, v_{2, y}\right), d_{G_{1} \circ G_{2}}\left(v_{2, x}, u_{2, y}\right),\right. \\
& \left.d_{G_{1} \circ G_{2}}\left(v_{2, x}, v_{2, y}\right)\right\} \\
= & \max \left\{d_{G_{1}}(x, y)+2, d_{G_{1}}(x, y)+2, d_{G_{1}}(x, y)+2, d_{G_{1}}(x, y)+2\right\} \\
= & d_{G_{1}}(x, y)+2 .
\end{aligned}
$$

Now,

$$
\begin{aligned}
W_{2} & =\sum_{\{g, f\} \subseteq S_{2}} d_{e \mid G_{1} \circ G_{2}}(g, f) \\
& =\sum_{x \in V\left(G_{1}\right)} \sum_{\{g, f\} \subseteq S_{2, x}} d_{e \mid G_{1} \circ G_{2}}(g, f)+\sum_{\{x, y\} \subseteq V\left(G_{1}\right)} \sum_{g \in S_{2, x}} \sum_{f \in S_{2, y}} d_{e \mid G_{1} \circ G_{2}}(g, f)
\end{aligned}
$$




$$
\begin{aligned}
& =\sum_{x \in V\left(G_{1}\right)}\left(2\left(\begin{array}{c}
e_{2} \\
2
\end{array}\right)-3\left(\Delta\left(G_{2}\right)+\Theta\left(G_{2}\right)\right)\right)+e_{2}^{2} \sum_{\{x, y\} \subseteq V\left(G_{1}\right)}\left(d_{G_{1}}(x, y)+2\right) \\
& =n_{1}\left(2\left(\begin{array}{c}
e_{2} \\
2
\end{array}\right)-3\left(\Delta\left(G_{2}\right)+3 \Theta\left(G_{2}\right)\right)\right)+e_{2}^{2}\left(W\left(G_{1}\right)+2\left(\begin{array}{c}
n_{1} \\
2
\end{array}\right)\right) .
\end{aligned}
$$

Case 3. $\{g, f\} \subseteq S_{3}, g \in S_{3, x}$ and $f \in S_{3, y}$.

If $x=y$ and $g=u_{2, x} x, f=v_{2, x} x$ where $u_{2, x}, v_{2, x} \in V\left(G_{2, x}\right)$, then

$$
\begin{aligned}
d_{e \mid G_{1} \circ G_{2}}(g, f)= & \max \left\{d_{G_{1} \circ G_{2}}\left(u_{2, x}, v_{2, x}\right), d_{G_{1} \circ G_{2}}\left(u_{2, x}, x\right), d_{G_{1} \circ G_{2}}\left(x, v_{2, x}\right),\right. \\
& \left.d_{G_{1} \circ G_{2}}(x, x)\right\} \\
= & \max \left\{d_{G_{1} \circ G_{2}}\left(u_{2, x}, v_{2, x}\right), 1,1,0\right\} .
\end{aligned}
$$

If $v_{2, x}$ is adjacent to $u_{2, x}$ in $G_{2, x}$, then $d_{G_{1} \circ G_{2}}\left(u_{2, x}, v_{2, x}\right)=1$, so $d_{e \mid G_{1} \circ G_{2}}(g, f)=$ 1 , otherwise $d\left(u_{2, x}, v_{2, x}\right)=2$, so $d_{e \mid G_{1} \circ G_{2}}(g, f)=2$. Hence for each edge $g=$ $u_{2, x} x \in S_{3, x}, \operatorname{deg}_{G_{2, x}}\left(u_{2, x}\right)$ edges of $S_{3, x}$ are at distance 1 from $g$ and all other edges are at distance 2 . So

$$
\begin{aligned}
& \sum_{\{g, f\} \subseteq S_{3, x}} d_{e \mid G_{1} \circ G_{2}}(g, f) \\
= & \frac{1}{2} \sum_{u_{2, x} \in V\left(G_{2, x}\right)}\left(\operatorname{deg}_{G_{2, x}}\left(u_{2, x}\right)+2\left(n_{2}-1-\operatorname{deg}_{G_{2, x}}\left(u_{2, x}\right)\right)\right) \\
= & \frac{1}{2}\left(2 e_{2}+2 n_{2}\left(n_{2}-1\right)-4 e_{2}\right)=n_{2}\left(n_{2}-1\right)-e_{2} .
\end{aligned}
$$

If $x \neq y$ and $g=u_{2, x} x \in S_{3, x}, f=u_{2, y} y \in S_{3, y}$, then

$$
\begin{aligned}
d_{e \mid G_{1} \circ G_{2}}(g, f)= & \max \left\{d_{G_{1} \circ G_{2}}\left(u_{2, x}, u_{2, y}\right), d_{G_{1} \circ G_{2}}\left(u_{2, x}, y\right), d_{G_{1} \circ G_{2}}\left(x, u_{2, y}\right),\right. \\
& \left.d_{G_{1} \circ G_{2}}(x, y)\right\} \\
= & \max \left\{d_{G_{1}}(x, y)+2, d_{G_{1}}(x, y)+1, d_{G_{1}}(x, y)+1, d_{G_{1}}(x, y)\right\} \\
= & d_{G_{1}}(x, y)+2 .
\end{aligned}
$$

Now,

$$
\begin{aligned}
W_{3} & =\sum_{\{g, f\} \subseteq S_{3}} d_{e \mid G_{1} \circ G_{2}}(g, f) \\
& =\sum_{x \in V\left(G_{1}\right)} \sum_{\{g, f\} \subseteq S_{3, x}} d_{e \mid G_{1} \circ G_{2}}(g, f)+\sum_{\{x, y\} \subseteq V\left(G_{1}\right)} \sum_{g \in S_{3, x}} \sum_{f \in S_{3, y}} d_{e \mid G_{1} \circ G_{2}}(g, f) \\
& =\sum_{x \in V\left(G_{1}\right)}\left(n_{2}\left(n_{2}-1\right)-e_{2}\right)+\sum_{\{x, y\} \subseteq V\left(G_{1}\right)} \sum_{g \in S_{3, x}} \sum_{f \in S_{3, y}}\left(d_{G_{1}}(x, y)+2\right) \\
& =n_{1}\left(n_{2}\left(n_{2}-1\right)-e_{2}\right)+n_{2}^{2} \sum_{\{x, y\} \subseteq V\left(G_{1}\right)}\left(d_{G_{1}}(x, y)+2\right)
\end{aligned}
$$




$$
\begin{aligned}
& =n_{1}\left(n_{2}\left(n_{2}-1\right)-e_{2}\right)+n_{2}^{2} W\left(G_{1}\right)+2 n_{2}^{2}\left(\begin{array}{c}
n_{1} \\
2
\end{array}\right) \\
& =2\left(\begin{array}{c}
n_{1} n_{2} \\
2
\end{array}\right)-n_{1} e_{2}+n_{2}^{2} W\left(G_{1}\right) .
\end{aligned}
$$

Case 4. $g \in S_{1}, f \in S_{2}$.

Let $g=u_{1} v_{1} \in S_{1}, f=u_{2, x} v_{2, x} \in S_{2, x}$, for some $x \in V\left(G_{1}\right)$. Then

$$
\begin{aligned}
d_{e \mid G_{1} \circ G_{2}}(g, f)= & \max \left\{d_{G_{1} \circ G_{2}}\left(u_{1}, u_{2, x}\right), d_{G_{1} \circ G_{2}}\left(u_{1}, v_{2, x}\right), d_{G_{1} \circ G_{2}}\left(v_{1}, u_{2, x}\right),\right. \\
& \left.\quad d_{G_{1} \circ G_{2}}\left(v_{1}, v_{2, x}\right)\right\} \\
= & \max \left\{d_{G_{1}}\left(u_{1}, x\right)+1, d_{G_{1}}\left(u_{1}, x\right)+1, d_{G_{1}}\left(v_{1}, x\right)+1,\right. \\
& \left.\quad d_{G_{1}}\left(v_{1}, x\right)+1\right\} \\
= & \max \left\{d_{G_{1}}\left(u_{1}, x\right), d_{G_{1}}\left(v_{1}, x\right)\right\}+1 \\
= & D_{G_{1}}(x, g)+1 .
\end{aligned}
$$

Now,

$$
\begin{aligned}
W_{4} & =\sum_{x \in V\left(G_{1}\right)} \sum_{f \in S_{2, x}} \sum_{g \in S_{1}} d_{e \mid G_{1} \circ G_{2}}(g, f)=\sum_{x \in V\left(G_{1}\right)} \sum_{f \in S_{2, x}} \sum_{g \in S_{1}}\left(D_{G_{1}}(x, g)+1\right) \\
& =e_{2} W_{v e}\left(G_{1}\right)+n_{1} e_{1} e_{2}=e_{2}\left(W_{v e}\left(G_{1}\right)+n_{1} e_{1}\right) .
\end{aligned}
$$

Case 5. $g \in S_{1}, f \in S_{3}$

Let $g=u_{1} v_{1} \in S_{1}, f=u_{2, x} x \in S_{3, x}$, where $u_{1}, v_{1}, x \in V\left(G_{1}\right), u_{2, x} \in V\left(G_{2, x}\right)$.

Then

$$
\begin{aligned}
d_{e \mid G_{1} \circ G_{2}}(g, f)= & \max \left\{d_{G_{1} \circ G_{2}}\left(u_{1}, u_{2, x}\right), d_{G_{1} \circ G_{2}}\left(u_{1}, x\right), d_{G_{1} \circ G_{2}}\left(v_{1}, u_{2, x}\right),\right. \\
& \left.d_{G_{1} \circ G_{2}}\left(v_{1}, x\right)\right\} \\
= & \max \left\{d_{G_{1}}\left(u_{1}, x\right)+1, d_{G_{1}}\left(u_{1}, x\right), d_{G_{1}}\left(v_{1}, x\right)+1, d_{G_{1}}\left(v_{1}, x\right)\right\} \\
= & \max \left\{d_{G_{1}}\left(u_{1}, x\right), d_{G_{1}}\left(v_{1}, x\right)\right\}+1 \\
= & D_{G_{1}}(x, g)+1 .
\end{aligned}
$$

Now,

$$
\begin{aligned}
W_{5} & =\sum_{x \in V\left(G_{1}\right)} \sum_{f \in S_{3, x}} \sum_{g \in S_{1}} d_{e \mid G_{1} \circ G_{2}}(g, f)=\sum_{x \in V\left(G_{1}\right)} \sum_{f \in S_{3, x}} \sum_{g \in S_{1}}\left(D_{G_{1}}(x, g)+1\right) \\
& =n_{2} W_{v e}\left(G_{1}\right)+n_{1} e_{1} n_{2}=n_{2}\left(W_{v e}\left(G_{1}\right)+n_{1} e_{1}\right) .
\end{aligned}
$$

Case 6. $g \in S_{2}, f \in S_{3}$

Let $g=u_{2, x} v_{2, x} \in S_{2, x}, f=z_{2, x} x \in S_{3, x}$, where $x \in V\left(G_{1}\right)$ and $u_{2, x}, v_{2, x}, z_{2, x} \in$ $V\left(G_{2, x}\right)$. Then

$$
\begin{gathered}
d_{e \mid G_{1} \circ G_{2}}(g, f)=\max \left\{d_{G_{1} \circ G_{2}}\left(u_{2, x}, z_{2, x}\right), d_{G_{1} \circ G_{2}}\left(u_{2, x}, x\right), d_{G_{1} \circ G_{2}}\left(v_{2, x}, z_{2, x}\right),\right. \\
\left.d_{G_{1} \circ G_{2}}\left(v_{2, x}, x\right)\right\}
\end{gathered}
$$




$$
=\max \left\{d_{G_{1} \circ G_{2}}\left(u_{2, x}, z_{2, x}\right), 1, d_{G_{1} \circ G_{2}}\left(v_{2, x}, z_{2, x}\right), 1\right\} .
$$

By definition of $G_{1} \circ G_{2}$, the distances $d_{G_{1} \circ G_{2}}\left(u_{2, x}, z_{2, x}\right)$ and $d_{G_{1} \circ G_{2}}\left(v_{2, x}, z_{2, x}\right)$ are equal to 0,1 or 2 . It is easy to see that, if $z_{2, x}=u_{2, x}$ or $z_{2, x}=v_{2, x}$ or $u_{2, x} z_{2, x}, v_{2, x} z_{2, x} \in S_{2, x}$, then $d_{e \mid G_{1} \circ G_{2}}(g, f)=1$, otherwise $d_{e \mid G_{1} \circ G_{2}}(g, f)=2$. So, for each edge $g=u_{2, x} v_{2, x} \in S_{2, x}$, the edges $u_{2, x} x, v_{2, x} x, z_{2, x} x \in S_{3, x}$, where $z_{2, x} \in N_{G_{2, x}}\left(u_{2, x}\right) \cap N_{G_{2, x}}\left(v_{2, x}\right)$ are at distance 1 from $g$ and all other edges are at distance 2. So

$$
\begin{aligned}
& \sum_{g \in S_{2, x}} \sum_{f \in S_{3, x}} d_{e \mid G_{1} \circ G_{2}}(g, f) \\
& =\sum_{u_{2, x}}\left(2+\left|N_{v_{2, x} \in S_{2, x}}\left(u_{2, x}\right) \cap N_{G_{2, x}}\left(v_{2, x}\right)\right|\right. \\
& \left.+2\left(n_{2}-2-\left|N_{G_{2, x}}\left(u_{2, x}\right) \cap N_{G_{2, x}}\left(v_{2, x}\right)\right|\right)\right) \\
& =\sum_{u_{2, x} v_{2, x} \in S_{2, x}}\left(2 n_{2}-2-\left|N_{G_{2, x}}\left(u_{2, x}\right) \cap N_{G_{2, x}}\left(v_{2, x}\right)\right|\right)=2 e_{2}\left(n_{2}-1\right)-3 \Delta\left(G_{2}\right) . \\
& d_{e \mid G_{1} \circ G_{2}}(g, f)=\max \left\{d_{G_{1} \circ G_{2}}\left(u_{2, x}, u_{2, y}\right), d_{G_{1} \circ G_{2}}\left(u_{2, x}, y\right), d_{G_{1} \circ G_{2}}\left(v_{2, x}, u_{2, y}\right)\right. \text {, } \\
& \left.d_{G_{1} \circ G_{2}}\left(v_{2, x}, y\right)\right\} \\
& =\max \left\{d_{G_{1}}(x, y)+2, d_{G_{1}}(x, y)+1, d_{G_{1}}(x, y)+2, d_{G_{1}}(x, y)+1\right\} \\
& =d_{G_{1}}(x, y)+2 \text {. }
\end{aligned}
$$

Now,

$$
\begin{aligned}
W_{6} & =\sum_{x, y \in V\left(G_{1}\right)} \sum_{g \in S_{2, x}} \sum_{f \in S_{3, y}} d_{e \mid G_{1} \circ G_{2}}(g, f) \\
& =\sum_{x \in V\left(G_{1}\right)} \sum_{g \in S_{2, x}} \sum_{f \in S_{3, x}} d_{e \mid G_{1} \circ G_{2}}(g, f) \\
& +\sum_{x \in V\left(G_{1}\right)} \sum_{y \in V\left(G_{1}\right) \backslash\{x\}} \sum_{g \in S_{2, x}} \sum_{f \in S_{3, y}} d_{e \mid G_{1} \circ G_{2}}(g, f) \\
& =\sum_{x \in V\left(G_{1}\right)}\left(2 e_{2}\left(n_{2}-1\right)-3 \Delta\left(G_{2}\right)\right) \\
& +\sum_{x \in V\left(G_{1}\right)} \sum_{y \in V\left(G_{1}\right) \backslash\{x\}} \sum_{g \in S_{2, x}} \sum_{f \in S_{3, y}}\left(d_{G_{1}}(x, y)+2\right) \\
& =n_{1}\left(2 e_{2}\left(n_{2}-1\right)-3 \Delta\left(G_{2}\right)\right)+n_{2} e_{2} \sum_{x \in V\left(G_{1}\right)} \sum_{y \in V\left(G_{1}\right) \backslash\{x\}}\left(d_{G_{1}}(x, y)+2\right) \\
& =n_{1}\left(2 e_{2}\left(n_{2}-1\right)-3 \Delta\left(G_{2}\right)\right)+2 n_{2} e_{2}\left(W\left(G_{1}\right)+n_{1}\left(n_{1}-1\right)\right) .
\end{aligned}
$$


Now the formula for the edge-Wiener index of $G_{1} \circ G_{2}$ follows by adding all six contributions and simplifying the resulting expression.

Again, it is interesting to note that the formula of Theorem 2 does not include any invariants of $G_{2}$ that depend on its connectivity. It is, hence, possible to apply Theorem 2 to corona products $G_{1} \circ G_{2}$ with disconnected $G_{2}$.

\section{EXAMPLES AND COROLLARIES}

Now, we can obtain explicit formulas for the edge-Wiener indices of some classes of graphs by specializing components in joins and coronas. We start by computing the edge Wiener-index of a suspension of a graph $G$. For a given graph $G$, we call the graph $K_{1}+G$ the suspension of $G$, where $K_{1}$ denotes the single vertex graph.

Corollary 1. Let $G$ be a simple graph of order $n$ and size e. Then

$$
W_{e}\left(K_{1}+G\right)=W_{e}\left(K_{1} \circ G\right)=2\left(\begin{array}{c}
n+e \\
2
\end{array}\right)-3 e-6 \Delta(G)-3 \Theta(G) .
$$

Now the formulas for the wheel graph $W_{n}=K_{1}+C_{n}$ and for the fan graph $K_{1}+P_{n}$ follow at once. Both graphs allow alternative representations as $K_{1} \circ C_{n}$ and $K_{1} \circ P_{n}$, respectively.

\section{Corollary 2.}

$$
\begin{aligned}
& W_{e}\left(K_{1}+C_{n}\right)=\left\{\begin{array}{ll}
15 & n=3 \\
4 n^{2}-5 n & n \geq 4
\end{array} ;\right. \\
& W_{e}\left(K_{1}+P_{n}\right)=4 n^{2}-9 n+5, \quad n \geq 2 .
\end{aligned}
$$

Our next example is the windmill graph. The windmill graph $D_{n}^{(m)}$ is the graph obtained by taking $m$ copies of the complete graph $K_{n}$ with a vertex in common. The case $n=3$ therefore corresponds to the Dutch windmill graph. One can easily see that the windmill graph is the suspension of $m$ copies of $K_{n-1}$. Also, it is easy to see that, $\Delta\left(K_{n}\right)=\left(\begin{array}{l}n \\ 3\end{array}\right)$ and $\Theta\left(K_{n}\right)=\left(\begin{array}{c}n \\ 4\end{array}\right)$, for $n \geq 1$. So using Corollary 1 , we can get the formula for the edge-Wiener index of the windmill graph $D_{n}^{(m)}$.

Corollary 3. For $n \geq 2$ and $m \geq 1$,

$$
W_{e}\left(D_{n}^{(m)}\right)=\frac{1}{4} m\left(\begin{array}{l}
n \\
2
\end{array}\right)\left(4 m\left(\begin{array}{l}
n \\
2
\end{array}\right)-n^{2}+n-2\right) .
$$
by:

In particular, the edge-Wiener index of the Dutch windmill graph $D_{3}^{(m)}$ is given

Corollary 4. For $m \geq 1$,

$$
W_{e}\left(D_{3}^{(m)}\right)=3 m(3 m-2) .
$$


Now, we turn our attention toward coronas. Coronas sometimes appear in chemical literature as plerographs of the usual hydrogen-suppressed molecular graphs known as kenographs; see [13] for definitions and more information. The $t$-thorny graph of a given graph $G$ is obtained as $G \circ \bar{K}_{t}$, where $\bar{K}_{t}$ denotes the empty graph on $t$ vertices [11]. For the $t$-thorny graph of a graph $G$, we obtain the following formula.

Corollary 5. Let $G$ be a simple connected graph of order $n$ and size e. Then

$$
W_{e}\left(G \circ \bar{K}_{t}\right)=W_{e}(G)+t^{2} W(G)+t W_{v e}(G)+n t(n t+e-1) .
$$

Now, we present two formulas for the edge-Wiener indices of the $t$-thorny cycle $C_{n} \circ \bar{K}_{t}$ and the $t$-thorny path $P_{n} \circ \bar{K}_{t}$. We use known results for the edge-Wiener indices of paths and cycles [9] and our results on the vertex-edge Wiener indices of these graphs obtained in [1].

\section{Corollary 6.}

$$
\begin{gathered}
W_{e}\left(C_{n} \circ \bar{K}_{t}\right)=\left\{\begin{array}{ll}
\frac{n(t+1)}{8}\left[n^{2}(t+1)+4 n(2 t+1)-(t+13)\right] & n \text { is odd } \\
\frac{n(t+1)}{8}\left[n^{2}(t+1)+4 n(2 t+1)-8\right] & n \text { is even }
\end{array} ;\right. \\
W_{e}\left(P_{n} \circ \bar{K}_{t}\right)=\left(\begin{array}{c}
n+1 \\
3
\end{array}\right)(t+1)^{2}+(n t+n-1)(n t-1) .
\end{gathered}
$$

Interesting classes of graphs can also be obtained by specializing the first component in the corona product. For example, for a graph $G$, the graph $K_{2} \circ G$ is called its bottleneck graph. For the bottleneck graph of a graph $G$, we obtain the following formula.

Corollary 7. Let $G$ be a simple graph of order $n$ and size $e$. Then

$$
W_{e}\left(K_{2} \circ G\right)=5(n+e)^{2}+2(n-2 e)-12 \Delta(G)-6 \Theta(G) .
$$

Using Corollary 7, the formulas for the bottleneck graph of a cycle $K_{2} \circ C_{n}$ and the bottleneck graph of a path $K_{2} \circ P_{n}$ are easily obtained.

\section{Corollary 8.}

$$
\begin{gathered}
W_{e}\left(K_{2} \circ C_{n}\right)=\left\{\begin{array}{ll}
162 & n=3 \\
20 n^{2}-2 n & n \geq 4
\end{array} ;\right. \\
W_{e}\left(K_{2} \circ P_{n}\right)=20 n^{2}-22 n+9 .
\end{gathered}
$$

\section{ACKNOWLEDGEMENT}

The authors would like to thank the referee for his/her careful reading and useful suggestions which led us to improve the paper. 


\section{REFERENCES}

[1] M. Azari and A. Iranmanesh, "Computation of the edge wiener indices of the sum of graphs," Ars Combin., vol. 100, pp. 113-128, 2011.

[2] M. Azari, A. Iranmanesh, and A. Tehranian, "Computation of the first edge wiener index of a composition of graphs," Studia Univ. Babes Bolyai Chem., no. 4, pp. 183-196, 2010.

[3] M. Azari, A. Iranmanesh, and A. Tehranian, "Maximum and minimum polynomials of a composite graph,” Austral. J. Basic Appl. Sci., vol. 5, no. 9, pp. 825-830, 2011.

[4] M. Azari, A. Iranmanesh, and A. Tehranian, "A method for calculating an edge version of the wiener number of a graph operation," Util. Math., vol. 87, pp. 151-164, 2012.

[5] M. Azari, A. Iranmanesh, and A. Tehranian, "Two topological indices of three chemical structures," MATCH Commun. Math. Comput. Chem., vol. 69, no. 1, pp. 69-86, 2013.

[6] P. Dankelmann, I. Gutman, S. Mukwembi, and H. C. Swart, "The edge wiener index of a graph," Discrete Math., vol. 309, no. 10, pp. 3452-3457, 2009.

[7] I. Gutman, "Calculating the wiener number: the doyle-graver method," J. Serb. Chem. Soc., vol. 58, pp. 745-750, 1993.

[8] I. Gutman, Y. N. Yeh, S. L. Lee, and Y. L. Luo, "Some recent results in the theory of the wiener number," Indian J. Chem., vol. 32A, pp. 651-661, 1993.

[9] A. Iranmanesh, I. Gutman, O. Khormali, and A. Mahmiani, "The edge versions of wiener index," MATCH Commun. Math. Compute. Chem., vol. 61, no. 3, pp. 663-672, 2009.

[10] M. H. Khalifeh, H. Yousefi-Azari, A. R. Ashrafi, and S. G. Wagner, "Some new results on distancebased graph invariants," European J. Combin., vol. 30, no. 5, pp. 1149-1163, 2009.

[11] D. J. Klein, T. Došlić, and D. Bonchev, "Vertex-weightings for distance moments and thorny graphs," Discrete Appl. Math., vol. 155, no. 17, pp. 2294-2302, 2007.

[12] H. Liu and X. F. Pan, "On the wiener index of trees with fixed diameter," MATCH Commun. Math. Comput. Chem., vol. 60, no. 1, pp. 85-94, 2008.

[13] A. Miličević and N. Trinajstić, "Combinatorial enumeration in chemistry," in Chemical Modelling: Applications and Theory, A. Hincliffe, Ed. Cambridge: RSC Publishing, 2006, pp. 405-469.

[14] N. Trinajstić, Chemical Graph Theory. Boca Raton: CRC Press, 1992.

[15] S. G. Wagner, "A class of trees and its wiener index," Acta Appl. Math., vol. 91, no. 2, pp. 119-132, 2006.

[16] H. Wiener, "Correlation of heats of isomerization and differences in heats of vaporization of isomers among the paraffin hydrocarbons," J. Am. Chem. Soc., vol. 69, no. 11, pp. 2636-2638, 1947.

[17] H. Wiener, "Structural determination of paraffin boiling points," J. Am. Chem. Soc., vol. 69, no. 1, pp. 17-20, 1947.

Authors' addresses

M. Azari

Department of Mathematics, Kazerun Branch, Islamic Azad University, P. O. Box: 73135-168, Kazerun, Iran

E-mail address: azari@kau.ac.ir

\section{A. Iranmanesh}

Department of pure Mathematics, Faculty of Mathematical Sciences, Tarbiat Modares University, P.

O. Box: 14115-137, Tehran, Iran

E-mail address: iranmaneshemodares.ac.ir 